\title{
11. The Procedure Division
}

The program instructions are written in the Procedure Division - organised in a similar way to a book or a report written in English.

The Procedure Division may be divided into Sections - each with a heading; these are approximately equivalent to chapters in a book. Just as you would not generally have chapters in a short essay - a short program will not usually have sections.

The program is further divided into paragraphs; these would correspond to the paragraphs in an essay or report.

Each paragraph must be given a suitable heading (see lesson 7 for permissible names), and must finish with a full-stop.

Always divide your program into paragraphs (if you are using sections - then these should be split into paragraphs).

Although it is possible to divide a paragraph into sentences (and this is usual and necessary if you are using a style based on COBOL 74) each one ending with a full-stop - it is advisable not to do this if you are using the structured constructs of COBOL 85 suggested in this book, as errors can easily result unless you fully understand both programming styles.

A paragraph generally contains one or more program statements - each one an instruction to carry out a single task. A paragraph may, if required, be empty (i.e. just a heading).

A statement consists of one or more $\mathrm{COBOL}$ command words (sometimes called Verbs) - e.g. DISPLAY, COMPUTE, ACCEPT - together with additional information, such as datanames.

\section{Exercise}

1. Give some examples of permitted paragraph names (see lesson 7).

2. Give 4 examples of:

a) statements;

b) paragraphs.

3. Give one advantage of splitting a program into headed Sections or Paragraphs.

4. Give three examples of $\mathrm{COBOL}$ statements (look at the example programs on lessons 5 and 6 ).

5. Place the following in the correct order (from large to small): statement, section, division, paragraph. 